\title{
Size matters: Finding growth pathways that protect the heart
}

Cell Research (2017) 27:1187-1188. doi:10.1038/cr.2017.120; published online 19 September 2017

In a recent paper published in Cell Research, Abdul-Ghani and colleagues show that the cytokine, cardiotrophin-1 (CT1), drives a protective form of reversible cardiac hypertrophy that acts through a nonapoptotic caspase-dependent mechanism. Since CT1 can be delivered as exogenous protein, these studies provide new biological insights and potential translational opportunities.

The mammalian heart responds to stress through well-studied adaptations, including an increase in heart mass, termed cardiac hypertrophy. Cardiac hypertrophy is often broadly categorized as pathological or physiological. The increased heart mass seen in both is primarily driven by increased cardiomyocyte size, although an increase in cell number may contribute modestly in some settings. Pathological growth occurs in response to pressure overload states like hypertension or aortic stenosis, and often leads to adverse outcomes including heart failure. In contrast, physiological growth occurs during postnatal development, pregnancy, and after sustained exercise training. While there are likely important differences among physiological growth states, in general they do not lead to adverse sequelae and can even be cardioprotective.

Cardiotrophin-1 (CT1) is a cytokine in the interleukin-6 family that signals through gp130-dependent pathways and is known to induce cardiac hypertrophy [1]. Initially, CT1 was thought to drive pathological hypertrophy but was found to promote cell survival and induce proliferation of embryonic cardiomyocytes [2], creating interest in its cardioprotective effects. In a recent paper in Cell Research, Abdul-Ghani et al. [3] show that CT1 causes a reversible, protective form of cardiomyocyte hypertrophy. These effects are dependent on transient and incomplete activation of the caspase cascade, the activation of which is attenuated by casein kinase 2 (CK2), a kinase known to modulate cardiac hypertrophy. CT1 also enhanced myocardial vascularity and curtailed the development of right heart failure in a model of pulmonary hypertension. Finally, CT1 improved cardiac function after LAD ligation.

This interesting work underscores several questions central to the field:

What distinguishes physiological from pathological hypertrophy? Transcriptional profiles in early pathological and physiological hypertrophy are largely distinct [4], as are the dominant protein regulators of these states. While there are pathways activated in both, some of these play dramatically different roles in each; for example, Akt1 is necessary for exercise-induced physiological growth but inhibits pathological hypertrophy [5]. These observations highlight the crosstalk and potential for reciprocal compensation between physiological and pathological cardiac growth. Importantly, this dichotomous classification of hypertrophy as either pathological or physiological is undoubtedly an oversimplification, particularly when we include states induced by specific molecular interventions devoid of their usual pathophysiological context. The current study employing pharma- cological delivery of CT1 may be a case in point. Not all the phenotypes documented by Abdul-Ghani and colleagues fall neatly into one category or the other. For example, the authors confirmed that CT1 increases the length-width ratios of cells, in contrast to the pattern seen with phenylephrine, which they use as a pathological stimulus. In fact, cardiomyocyte elongation is seen in pathological hypertrophy and notable physiological growth pathways (e.g., neuregulin-1 and CITED4) inhibit elongation [6]. Moreover, phenylephrine itself likely also activates physiological growth pathways given the role of $\alpha$-adrenergic signaling in postnatal cardiac growth [7]. The authors highlight the reversibility of CT1-induced growth as a physiological hallmark, although reversal can also be seen in pathological states. Finally, CT1 induced an increase in ANP expression, which the authors note can be seen with physiological hypertrophy although natriuretic peptide expression is more closely associated with pathology. Thus, it remains unclear whether CT1 truly induces physiological hypertrophy or at the dose and duration used, a compensated hypertrophy that has not progressed to frank pathology. Given Laplace's Law, early pathological hypertrophy can also mitigate dysfunction by reducing wall stress. Molecular profiling perhaps already available in their microarrays could help place CT1-induced hypertrophy on the physiological to pathological spectrum but to some extent this would be a semantic exercise.

Does the nature of the stress stimulus or its duration determine whether the 
heart mounts a pathological or physiological response? Abdul-Ghani et al. [3] hypothesize that CT1 transiently affects caspase signaling leading to physiological growth while longer activation would generate a pathological effect. Indeed, most pathological hypertrophic stimuli are sustained and chronic, while exercise is intermittent. Perrino and colleagues uncoupled stress type from duration using a model of intermittent thoracic aortic constriction (iTAC) [8]. iTAC led to pathological features not seen in duration-matched exercised hearts, suggesting that stimulus type was a dominant driver of phenotype. It seems likely that stimulus nature and 'dose' both contribute to outcome, and their relative contributions may vary with context.

What are the key mechanisms regulating cardiac hypertrophy? The current study highlights an unconventional role for caspases in hypertrophy that does not involve apoptosis. By limiting the

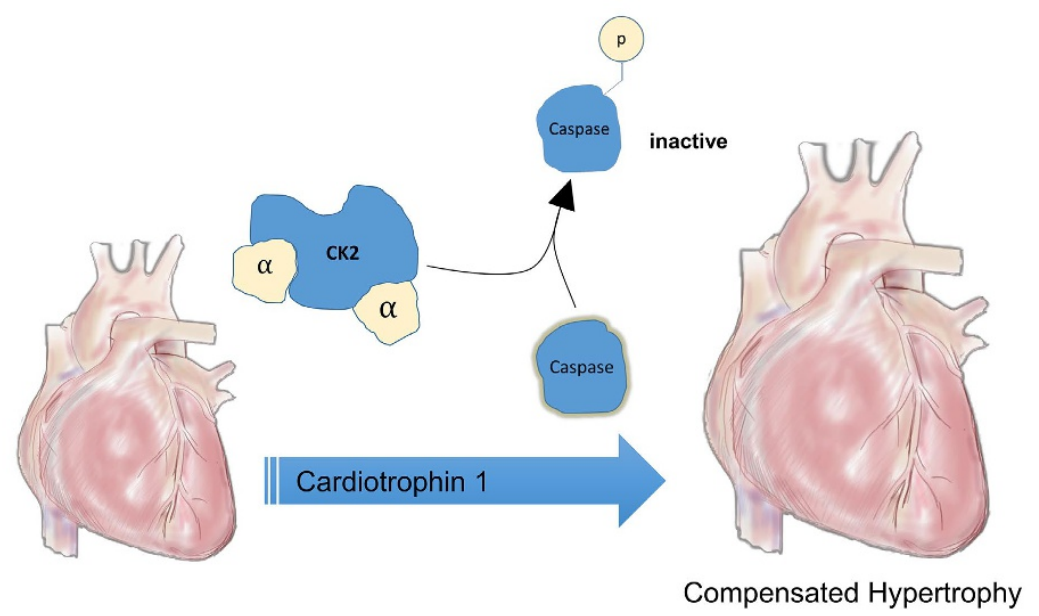

Figure 1 Exogenous CT1 induces caspase-dependent cardiac hypertrophy. CK2 limits caspase activation, which may prevent development of irreversible pathological hypertrophy. duration and degree of caspase activation, CK2 appears central to CT1's nuanced prohypertrophic effects [9], which also involve transient induction of two transcription factors long-recognized to mediate hypertrophy, MEF2 and NFKB. CK2 regulates caspase signaling by phosphorylating both caspase-3 and its targets to inhibit proteolytic cleavage (Figure 1), thus limiting complete execution of the caspase cascade. Of note, CK2 has previously been shown to promote cardiac growth by inhibiting p27, a cell cycle inhibitor [9]. p27 is highly expressed in the heart and downregulated in heart failure. The p27-knockout mouse has physiological hypertrophy at baseline with notable cardiomyocyte hyperplasia [9], but does worse after pressure overload. Interestingly, miR-222, which is necessary for exercise-induced physiological cardiac hypertrophy [10], also targets p27. Although neither p27 nor cell cycle activity was examined in the current study, it would be interesting to know whether CT1 treatment also resulted in CK2-mediated p27 inhibition that could contribute to increased cardiac mass. If so, inhibiting this tumor suppressor systemically could have adverse consequences.

While a more complete understanding of CT1's local and systemic effects will be essential in considering the translational implications of this work, AbdulGhani et al. have contributed to some of the central ongoing conversations regarding cardiac hypertrophy. Their work helps to focus attention on novel molecular drivers of heart growth and the relationship between physiological and pathological hypertrophy, exploring whether these insights can be exploited to mitigate models of heart disease.

\section{J Sawalla Guseh ${ }^{1}$, Anthony Rosenzweig ${ }^{1}$}

${ }^{1}$ Cardiology Division, Massachusetts General Hospital and Harvard Medical School, Boston, Massachusetts 02115

Correspondence: Anthony Rosenzweig

E-mail: rosenzwe@helix.mgh.harvard.edu

\section{References}

1 Wollert KC, Taga T, Saito M, et al. J Biol Chem 1996; 271:9535-9545.

2 Sheng Z, Pennica D, Wood WI, et al. Development 1996; 122:419-428.

3 Abdul-Ghani M, Suen C, Jiang B, et al. Cell Res 2017; 27:1195-1215.

4 Bostrom P, Mann N, Wu J, et al. Cell 2010; 143:1072-1083.

5 DeBosch B, Treskov I, Lupu TS, et al. Circulation 2006; 113:2097-2104.

6 Ryall KA, Bezzerides VJ, Rosenzweig A, et al. J Mol Cell Cardiol 2014; 72:74-84.

7 O'Connell TD, Ishizaka S, Nakamur A, et al. J Clin Invest 2003; 111:1783-1791.

8 Perrino C, Naga Prasad SV, Mao L, et al. J Clin Invest 2006; 116:1547-1560.

9 Hauck L, Harms C, An J, et al. Nat Med 2008; 14:315-324.

10 Liu X, Xiao J, Zhu H, et al. Cell Metab 2015; 21:584-595. 